\title{
THE ROLE OF AUTONOMOUS AGENTS IN TRAINING SCHOOL PSYCHOLOGY GRADUATE STUDENTS
}

\author{
Rogerio Eduardo da Silva and Elise N. Hendricker \\ University of Houston-Victoria \\ 3007 N Ben Wilson st., Victoria-TX, USA
}

\begin{abstract}
Graduate training in helping professions, such as psychology, requires knowledge, skills, and competency in discipline specific knowledge, as well as interpersonal skills. School psychologists are unique professionals trained to work with children, families, and school staff in public schools. The profession requires critical communication competencies across all domains of practice, yet training programs remain stagnant in ways to teach, measure, and evaluate these competencies in innovative ways. Interdisciplinary collaboration between computer science and school psychology holds great promise in remediating these concerns and advancing training using technology. Specifically, mixed reality and artificial intelligence tools, such as an autonomous training agent (ATA), where students can practice verbal and non-verbal communication strategies during clinical situations has the ability to provide deliberate practice for students in the classroom setting prior to working with real clients.
\end{abstract}

\section{KEYWORDS}

School Psychology, Mixed Reality, Autonomous Agents, Artificial Intelligence

\section{INTRODUCTION}

School psychologists are licensed professionals who provide psychological support in schools for children ages 3-21. They are uniquely trained to play a vital role in mental health promotion, prevention, and early intervention of social, emotional, and behavioral concerns. The National Association of School Psychologists [NASP] (2020) outlines graduate training standards of school psychology practices, along with ten domains of practice in which school psychologists should exhibit knowledge and competency. NASP (2020) also notes core beliefs drive school psychological practice, such as using effective strategies and skills to help children, demonstrating knowledge and skills effective for professional practice, and ensuring knowledge and skills reflect human diversity, cultural awareness, and social justice.

Across all the roles of the school psychologist, whether it be mental health assessment, consultation, intervention, or prevention, is the embedded organizational principle of professional communication (NASP, 2020). School psychologists must collaborate with a variety of stakeholders and exhibit strong communication skills to elicit appropriate information from children and families. They must effectively share results to make educational decisions, and display supportive, empathic communication with students and families to support their needs.

While these training domains and professional practices are outlined, graduate training programs in School Psychology are given latitude as to how these domains, knowledge, skills, and competencies are taught within their coursework. Research in the field has identified that communication competencies are critical to effective school psychology practice (Erchul et al., 2014), yet little has been done to identify how to teach these competencies (Newell \& Newman, 2014). For example, when teaching graduate students how to effectively conduct a suicide risk assessment, an instructor may simulate the role of an adolescent to ensure the graduate student can display the proper knowledge, skills, and communication techniques. Graduate students may also be paired with one another to simulate real world experiences.

More recently, integrating technology into training experiences has been explored. Studies incorporating technology using a deliberate practice approach to improve school psychology graduate students' communication skills has shown great promise, while also building the self-efficacy of students within their 
professional interactions (Newman et al., 2021). Applications from computer science, specifically using autonomous agents and artificial intelligence, holds great promise in providing advanced training to prepare school psychology students for the plethora of skills they will need to be successful in their careers.

The goal of this project is to develop a mixed reality tool where a school psychology student should be able to interact, using natural language and gestures, with an autonomous training agent (ATA) specially trained to perform a specific role, acting as the client during a session.

\section{DESIGNING AN AUTONOMOUS TRAINING AGENT}

To design these autonomous training agents, we anticipate three levels of description will be necessary: embodiment, behavior, and interaction.

Embodiment has to do with describing how the agent will appear to the user, i.e., its physical appearance, movements that include facial expressions, lip synching, body posture, voice synthesis, etc. Behavior should focus on creating the decision-making process for the agent, i.e., to decide how to proper respond to the user's dialog and gestures while still playing a predefined role during the conversation. Interaction has to do with the technological framework to be used to provide such an interface that is easy to use (intuitive and natural).

The next sections describe our initial ideas for each one of these levels.

\subsection{Embodied Conversational Agents}

In computer science, autonomous agents can be understood as "computational systems that inhabit some complex, dynamic environment, sense and act autonomously in this environment, and by doing so realize a set of goals or tasks for which they are designed" (Maes, 1995). Although these agents can be applied to a variety of applications, we are interested in relying on these agents to create a virtual actor.

According to Iurgel and Marcos (2007), a virtual actor is "an analogy to a real actor, which autonomously, and by its independent interpretation of the situation, can perform its role according to a given script, as part of a story". Once more, according to the literature, virtual actors can be called by many different names depending on their characteristics, goals, and application: virtual humans, autonomous digital actors, or embodied conversational agents.

Embodied Conversational Agents (or ECA) (Cassell 2000) are characters capable of engaging in a face-to-face conversation autonomously deciding proper behaviors considering four main functions: emotional, personality, performative and conversational.

- Emotional function relates to the ability the agent should demonstrate of understanding human emotion (face, voice, speech intonation, etc.) and responding coherently.

- Personality function means the agent should demonstrate a unique pattern of thoughts, emotions, and behavior.

- Performative function represents the agent's ability to communicate believably with a human interlocutor verbally (words and intonation), non-verbally (gestures, gaze, posture), and para-verbally (sounds).

- Conversational function relates to being able to understand what is being said in a conversation. E.g., words, sentences, dialog structure, and semantics.

\subsection{Producing Autonomous Behavior}

There are basically three types of agents: reactive, deliberative, and hybrid. Reactive agents act by reflex, which means that they establish a direct correlation between input and output; deliberative agents act as if, for each input, the agent would be capable of some sort of analysis and planning before acting; hybrid agents process the characteristics of both reactive and deliberative agents.

One hypothesis we intend to explore during this project is the feasibility of implementing the ECA as an EBDI agent (Jiang et al, 2007; Thume and Silva, 2012). An EBDI agent (emotion-belief-desire-intention) is an example of hybrid agents that relies on two concurrent paths for deliberation: 
- The primary emotions path (reactive layer) act as "filters", adjusting the priority of beliefs, allowing agents to accelerate the decision-making process.

- The secondary emotions path (deliberative layer) is used to refine this decision when time permits.

\subsection{Mixed Reality Interface}

To allow these autonomous training actors to provide a more vivid and productive learning experience to the student, the following aspects must be achieved:

1. They must look and move as realistically as possible.

2. They must behave as similar to human behavior as possible.

3. The interaction with them must be intuitive and natural, much like in a real scenario as possible.

For the first aspect, there are already tools available in the market to create and animate digital humans ${ }^{1}$ that should provide a very realistic quality for the agent's embodiment. In terms of human behavior simulation, as mentioned in section 2.2, we are planning to explore EBDI agents as a means to convincingly mimic human behavior.

Regarding producing an intuitive and natural interface for the students to interact with the agents, our proposal is to develop a mixed reality interface.

Mixed reality is a blend of physical and digital words that allows users to interact with virtual and real-world objects simultaneously ${ }^{2}$. It results from the combination of users' actions, environment sensing (through camera, microphones, and other sensors), and computer-generated systems (virtual worlds) at the same time, thus enhancing the user's physical environment with virtual objects that are interactable in a similar fashion than other objects within the environment.

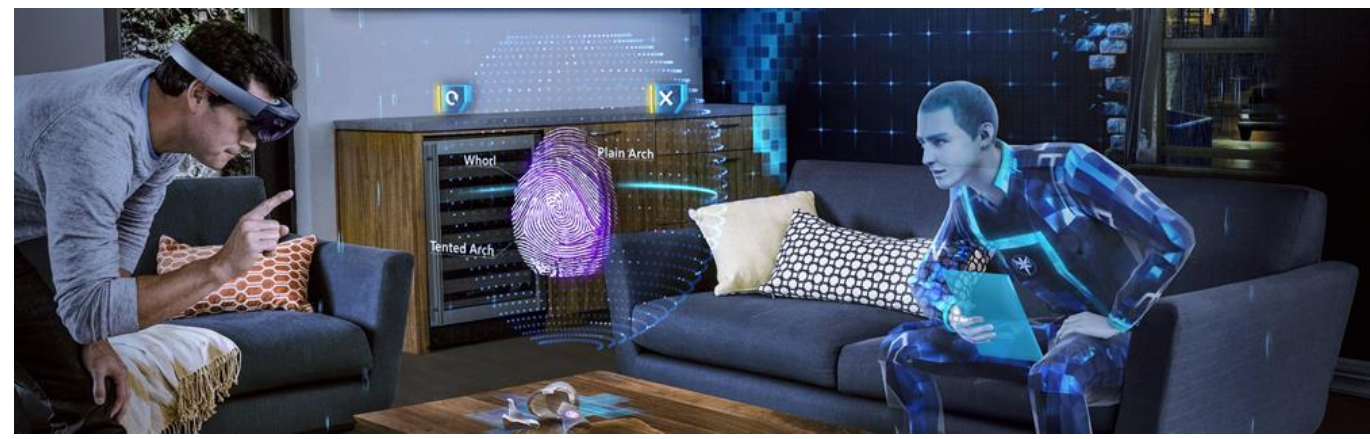

Figure 1. Example of mixed reality application using Microsoft Hololens

(Source: https://medium.com/hackernoon/the-dawn-of-mixed-reality-hololens-apps-9070a9800c26)

\section{FINAL REMARKS}

The proposed interdisciplinary project has clear advantages in advancing the training of school psychology graduate students. Communication skills, including verbal responses to clients, communication patterns, and understanding non-verbal behaviors, are often considered "soft skills" that are difficult to teach and practice, yet can play a large role in client outcomes in psychology disciplines. Advancing the use of technology to increase these skills can be beneficial in a number of helping fields (e.g., psychology, social work, and counseling) and other liberal arts and business disciplines (e.g., communication, marketing, and public relations).

\footnotetext{
${ }^{1}$ http://www.unrealengine.com/en-US/digital-humans

${ }^{2}$ https://docs.microsoft.com/en-us/windows/mixed-reality/discover/mixed-reality
} 


\section{REFERENCES}

Erchul, W. P., Grissom, P. F., Getty, K. C., \& Bennett, M. S. (2014). Researching interpersonal influence within school consultation: Social power base and relational communication perspectives. In W. P. Erchul \& S. M. Sheridan (Eds.), Handbook of research in school consultation (2nd ed., pp. 349-385). Routledge. https://doi.org/10.4324/9781315759623-24

Iurgel, I. A., and Marcos, A. F. (2007). Employing personality-rich virtual persons-New tools required. Computers \& Graphics 31, 6 (Dec.), 827-836.

Jiang, H., Vidal, J. M., and Huhns, M. N. (2007). EBDI: An architecture for emotional agents. In Proceedings of the Autonomous Agents and Multi-Agent Systems Conference, IEEE Computer Society.

Maes, P. (1995). Artificial life meets entertainment: Lifelike autonomous agents. Communications of the ACM, 38(11):108-114.

National Association of School Psychologists (2020). The Professional Standards of the National Association of School Psychologists. Author.

Newell, M., \& Newman, D. S. (2014). Assessing the state of evidence in consultation training: A review and call to the field. In W. P. Erchul \& S. M. Sheridan (Eds.), Handbook of research in school consultation (2nd ed., pp. 421-449). Routledge.

Newman, D. S., Gerrard, M. K., Villarreal, J. N., \& Kaiser, L. T. (2021). Deliberate practice of consultation microskills: An exploratory study of training. Training and Education in Professional Psychology. http://dx.doi.org/10.1037/tep0000368

Thume, G. S., and Silva, R.E. (2012). An Extended EBDI Model Applied to Autonomous Digital Actors, In: Brazilian Symposium on Games and Digital Entertainment (SBGAMES), Brasilia-DF, Brazil. 